\title{
NONEXISTENCE OF ASYMPTOTIC OBSERVABLES
}

\author{
JAMES S. HOWLAND ${ }^{1}$
}

Abstract. Strong asymptotic limits of all Heisenberg observables exist only for trivial Hamiltonians.

Lec $H$ be a selfadjoint operator on a separable Hilbert space $\mathscr{H}$. Lavine [1] has introduced into scattering theory the study of the algebra of bounded operators $A$ for which the strong asymptotic limits

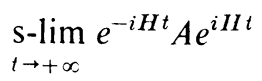

of the Heisenberg observables $A(t)=e^{-i H t} A e^{i I I t}$ exist. It is therefore of some interest that this algebra coincides with $\mathscr{B}(\mathscr{H})$ only in the trivial case.

THEOREM. The limit $(*)$ exists for every bounded $A$ iff $H$ is a constant multiple of the identity.

Proof. Suppose that $H$ is not a multiple of $I$. If $H$ has two distinct eigenvalues $\lambda$ and $\mu$ with eigenvectors $\phi$ and $\psi$, choose $A=(\cdot, \phi) \psi$. Then $A(t)=e^{i(\lambda-\mu) t}(\cdot, \phi) \psi$ has no limit. Otherwise, $H$ has a nontrivial continuous part, and since it suffices to construct $A$ on a reducing subspace of $H$, one may assume that $H$ is multiplication by $\lambda$ on $L_{2}([a, b), d g)$ where $g(\lambda)$ is a continuous increasing function with $g(a)=0$ and $g(b)=1$. If every interval $[\alpha, \beta)$ on which $g(\lambda)$ is constant is deleted from $[a, b)$, the remaining set supports $d g$ and is mapped by $g$ in a one-one measurepreserving fashion onto $[0,1)$ with Lebesgue measure. Under this change of variables, $H$ becomes multiplication by the strictly increasing function $\alpha(x)=g^{-1}(x)$ on $L_{2}[0,1)$. In this representation, choose $A f(x)=f(1-x)$, so that

$$
A(t) f(x)=e^{-i \beta(x) t} f(1-x)
$$

where $\beta(x)=\alpha(x)-\alpha .(1-x)$ is strictly increasing. Since

$$
\|A(t) f-A(s) f\|^{2}=\int_{0}^{1}\left|1-e^{i \beta(x)(t-s)}\right|^{2}|f(1-x)|^{2} d x
$$

Received by the editors November 8, 1971.

AMS 1970 subject classifications. Primary 47A40; Secondar! s1A4R.

${ }^{1}$ Supported by ARO Grant DA-ARO-D-31-124-G1005. 
which depends only on $t-s$, the strong limit can exist only if the right side vanishes for all $t$ and $s$. But since $\beta(x)$ is strictly increasing, this implies that $f=0$.

\section{REFERENCE}

1. R. B. Lavine, Scattering theory for long range potentials, J. Functional Analysis 5 (1970), 368-382. MR 42 \#5095.

Department of Mathematics, University of Virginia, Charlottesville, VirGINIA 22903 